\title{
Analyzing the Use of Ultrasound: Achilles Tendon Rupture
}

\author{
Massud Atta ${ }^{1}$, Shadi Jafari ${ }^{2}$, Kareen Moore $^{2}$ \\ ${ }^{1}$ Coliseum Medical Center, Macon, Georgia, USA \\ ${ }^{2}$ American University of Antigua, COM, Coolidge, Antigua
}

How to cite this paper: Atta, M., Jafari, S. and Moore, K. (2019) Analyzing the Use of Ultrasound: Achilles Tendon Rupture. Open Journal of Emergency Medicine, 7, 41-47.

https://doi.org/10.4236/ojem.2019.73005

Received: June 5, 2019

Accepted: August 10, 2019

Published: August 13, 2019

Copyright ( 2019 by author(s) and Scientific Research Publishing Inc. This work is licensed under the Creative Commons Attribution International License (CC BY 4.0).

http://creativecommons.org/licenses/by/4.0/

(c) (i) Open Access

\begin{abstract}
Growing evidence has made it obvious that early intervention in patients with Achilles tendon rupture extensively affects the prognosis. This requires the use of easily accessible imaging modalities such as ultrasound in establishing accurate diagnosis of tendinopathies so that early therapeutic decisions can be made. Ultrasound allows for assessment of tendons in a dynamic real time setting. Physicians can interact with patients and receive feedback regarding the symptomatic area, and assessing the tendon from different angles while under stress. It also offers a faster method to diagnose Achilles tendon rupture and therefore provide early intervention. Furthermore, ultrasound is a safe, non-invasive, and a patient friendly method that has become less expensive, portable, and a faster imaging modality to diagnose tendinopathies. In this paper, we review the application of ultrasound in diagnosing Achilles tendon rupture and comparing it with other imaging modalities, after thoroughly studying the current literature.
\end{abstract}

\section{Keywords}

Achilles Tendon Rupture, ATR, Ultrasound, Achilles Tendon, Tendon Rupture, Anisotropy, Compression Elastography, Gastrocnemius

\section{Introduction}

The Achilles tendon is the largest tendon in the human body [1]. With a mean length of approximately $15 \mathrm{~cm}$, it is formed by the union of the gastrocnemius and soleus muscles, and inserts posteriorly at the calcaneal bone [2]. The Achilles tendon is essential for flexion of the knee, as well as plantar flexion and inversion of the foot [3]. It is the most commonly injured tendon of the lower limbs [1]. The etiology for its injuries is often multifactorial [3]. Literature shows that age, male gender, and obesity are factors strongly correlated with patholo- 
gies of Achilles tendon [3] [4]. Other factors, including the use of Fluoroquinolone antibiotics and corticosteroids, have also been shown to lead to weakening of the tendon, with resulting Achilles tendinitis and increased risk of tendon rupture [3] [4].

Rupture of the Achilles tendon is most commonly sustained by competitive and recreational athletes, but can also occur in non-active individuals [4]. The peak age for rupture is $30-40$ years for both men and women, when the tendon begins to undergo degenerative changes [4]. "The incidence of Achilles tendon rupture (ATR) in the general population is approximately 5 to 10 per 100,000 , but may be higher in some regions and populations, and is increasing overall” [4]. Rupture is 4 to 5 times more common in men than in women [5]. "Over $80 \%$ of ruptures occur during recreational sports, and approximately $10 \%$ of patients who sustain an ATR have preexisting tendon pathologies [4]".

Tendon rupture occurs when sudden forces are exerted upon the Achilles tendon during strenuous physical activities that involve sudden pivoting on a foot or rapid acceleration [6]. Tendon rupture is more likely to occur where the blood supply of the gastrocnemius and soleus muscle complex is poorest, 2 - 6 $\mathrm{cm}$ above the insertion point [3] [4]. The injury is typically experienced as an audible snap followed by sudden calf pain, and difficulty with ambulation and weight bearing [6].

The diagnosis of Achilles tendon rupture can be made with a proper physical examination and history. The Thompson test, or squeezing of the calf muscle while the patient lies prone, can provide an accurate means for detecting complete Achilles tendon rupture [4]. In a completely ruptured tendon, a gap can be palpated usually $1-5 \mathrm{~cm}$ proximal to the calcaneal attachment [2]. While tendon rupture may be diagnosed solely by clinical examination, imaging modalities such as Ultrasonography (US) allow for rapid, non-invasive bedside confirmation of the diagnosis [5] [7].

Ultrasonography utilizes reflected pulses of high frequency waves to assess musculoskeletal structures. The Achilles tendon can best be visualized using a US probe with a frequency between 7.5 and $13 \mathrm{MHz}$ [5]. Both partial and complete tendon ruptures can be assessed with a US [4]. A normal Achilles tendon appears as a "ribbon-like, hypoechoic, tightly packed fibrillar structure" on US [8]. In tendon rupture, assessment with a US can demonstrate the presence of "hypoechogenicity with separation of the fibrillar structure or neovascularization by Doppler flow" [8].

\section{Uses of Ultrasound}

There are many different subtypes of ultrasound and they can be used in various ways to image an abnormality. A "Diagnostic Ultrasound" is used to image inside the body and has two types: Functional and Anatomical. Functional displays information such as tissue movement or velocity (ie. blood flow) while anatomical ultrasound takes assess internal organs and structures [9]. Therapeutic ul- 
trasound uses sound waves to manipulate tissues. This can be used for destroying stones in patients suffering from nephrolithiasis or even to dissolve clots [9].

Ultrasonography uses sound waves that penetrate into the tissue using a transducer that return to the ultrasound device which produces an image on screen. Piezoelectric crystals help to produce sound waves when an electric field is applied to them [9]. The sound waves transmit through tissue to the target area. The return echo is received by the transducer which generates electrical signals to the scanner and is displayed on a monitor screen. The speed of sound is used to calculate the distance between tissues on screen. A gel is used to prevent air pockets from forming and allows for better quality images [9].

When using an ultrasound to assess an ATR the patient should be lying prone and the transducer is placed along the longitudinal axis (sagittal plane) to evaluate the tendon. Then the transducer is rotated $90^{\circ}$ to evaluate the tendon on the transverse plane [10]. In a true ATR, one of the features seen on ultrasound is decreased vascularity $2-6 \mathrm{~cm}$ from the calcaneal insertion. Hypo-echogenicity would be seen in cases of partial-thickness tears of the Achilles tendon, while anechogenicity of the tendon fibers can be observed in some of the cases [10]. On ultrasound a complete tendon tear would show complete disruption of tendon fibers [10]. In addition, tendon retraction would be present along with a positive Thompson sign, where the foot no longer plantar flexes normally upon squeezing of the gastrocnemius muscle.

Ultrasound has been widely studied yet results vary between research papers. An article, by Kayser et al. mentions that ultrasound is useful for proximal tendon injuries but not for proximal ruptures which would require an MRI [11]. However, because of the varied usage of ultrasound such as compression elastography (CE) and ultrasound elastography, among others, ultrasound still plays a vital role in diagnosis and imaging of Achilles tendinopathies. For example, the compression ultrasound is used to compress the target tissue, assessing the fibers' strength. Softer tissues will be more deformed and the software will detect them with different colors: Red for soft tissue, blue for stiff tissue and yellow/green for intermediate tissue strength [12]. Another example is Ultrasound elastography (USE) which is useful for early diagnosis, degree of injury and tracking post-surgical follow up. In addition, USE can be used to screen athletes to prevent injury by modifying their training methods [12].

\section{Advantages of Ultrasound}

Ankle imaging is frequently being ordered to assess the pathologies of its tendons and ligaments [13] [14]. Different studies have researched the benefits of ultrasound and MRI in assessing ankle and foot tendon tears [15]. Ultrasound is sensitive and specific for detecting most cases of tendon tears whereas MRI is sensitive for detecting tears of larger tendons, such as Achilles tendon [8] [15]. Ultrasound is also able to differentiate partial from full thickness tears in Achilles tendon [15]. 
Ultrasound has several advantages compared to MRI in assessing tendons. Ultrasound allows for assessing tendons in a more dynamic real time setting [15]. The physician can interact with patients and receive feedback regarding the symptomatic area, assessing the tendon from different angles while under stress [15] [16] [17]. It also has the ability to evaluate the changes in tendon length during contraction [18]. For example, a complete Achilles tendon tear may be "poorly demarcated" due to the formation of a hematoma, however it can be better visualized during dorsiflexion of the ankle [16]. Ultrasound also offers a faster method to diagnose ATR and therefore provide early intervention [16]. With the help of doppler ultrasound, physicians can also assess "neovascularization in tendinopathy" [15]. Furthermore, ultrasound is a safe, non-invasive, and a patient friendly method that has become less expensive, portable, and a faster imaging modality to diagnose tendinopathies [16] [17] [19].

Compared to ultrasound, MRI is more expensive, not patient friendly and not readily available. It is also contraindicated in some patients with metal hardware. MRI cannot be performed in a dynamic setting and no feedback can be reported to the operator. Any movement during an MRI can distort the image quality [16]. Also, in MRI, imaging large areas are subjected to detection of abnormalities that may not be related to the chief complaint [16]. Other imaging modalities such has CT scan and fluoroscopy require ionizing radiation [16]. Patients with contrast induced allergy require prophylactic medication and those with kidney dysfunction are contraindicated from exposure to contrast [16].

In fact, X-ray may also be used when evaluating Achilles tendinopathies specifically looking at Kager's Triangle, a fat pad bordered by Flexor Hallucis longus (anteriorly), Achilles tendon (posteriorly) and calcaneus (inferiorly). A disruption in the Kager's triangle represents a possible Achilles tendon rupture. However, according to Kalebo et al. X-ray only shows soft tissue swelling (along with bony images) as opposed to ultrasound which can image the actual tendon [20].

\section{Disadvantage of Ultrasound}

While ultrasound offers some advantages over other modalities, it also has some disadvantages to be discussed. Ultrasound requires an acoustic window that is not needed when using MRI. Ultrasound beams are not able to penetrate through bony tissues [15]. Therefore, tendons and ligaments that are placed behind bones are more difficult to visualize [15]. Additionally, due to the stiff structure of tendons, ultrasound requires high frequency transducers to assess the integrity of tendons [15]. On the other hand, due to a strong magnetic field, MRI provides higher resolution imaging [15].

Penetrance of ultrasound waves is inversely proportional to the frequency of waves [16]. Higher frequency waves have lower penetrance while those with lower frequency penetrate deeper. Therefore, body habitus can be a limitation with the use of ultrasound. Recording of deeper structures such as tendons that 
need high frequency waves becomes limited in obese or muscular patients [16]. On the other hand, lower frequency has higher penetrance but a lower resolution and therefore makes the use of ultrasound more challenging in certain body habitus [16].

In contrast to other modalities, ultrasound necessitates a highly skilled operator [11] [17]. For example, fresh tears especially those of distal ruptures are easy to detect by experienced physicians [11]. Factors such as alignment of transducers, pressure applied to an area, and the knowledge of anatomy and pathology of a certain body parts contribute to the quality of the image recorded [19]. For these various reasons, operators are the great source of variability in the ultrasound images produced. Aside from a skilled operator, ultrasound devices with advanced equipment, high quality transducers and advanced softwares are more desired to record high resolution imaging [21].

Another factor that makes the use of ultrasound challenging for assessing tendinopathies is the concept of Anisotropy. Tendons normally appear echogenic on ultrasound. Highly structured tissues such as tendons and ligaments reflect sound beams depending on the angle that the beam has been applied to the tissue [13]. For example, to assess tendons, the ultrasound beam should be applied perpendicular to the tissue. Beams permeating in non-right angles can record the tendons as artificially hypoechoic and therefore mimic a tendinopathy [13]. In non-perpendicular angles, the degree of reflection of sound waves are reduced and therefore a hypoechogenic image is recorded [13] [16]. Hence a normal tendon can mimic a pathology due to the presence of artifacts (anisotropy) [13] [16].

\section{Discussion}

Research studies have shown different results regarding the efficacy of ultrasound and MRI in diagnosing Achilles tendinopathies [11] [15]. We found that there are advantages in using ultrasound to diagnose ATR compared to using MRI or other modalities. When ultrasound is used with a skilled technician, diagnosis can be achieved accurately in a short period of time. As mentioned by Liu et al. ultrasound has a sensitivity of $98 \%$ in diagnosing ATR with an accuracy of $93 \%$ [22]. In addition, ultrasound is portable and more accessible when compared to MRI. Lastly, MRI may display a higher quality image of ATR, but the efficiency, speed, low cost, less invasive and more efficient modality of ultrasound make it a better first option to evaluate ATR.

The main factor in choosing what imaging study to use to diagnose ATR also relates to its management and plan. Whether a patient requires surgical or non-surgical intervention relies on diagnostic findings. Surgical intervention has an increased risk of infection and nerve damage while non-surgical treatment can increase the risk of rerupture [23]. Therefore, it is important to decide on the most appropriate treatment based on clinical, physical and imaging findings. A history of an audible snap and sudden onset calf pain, as well as a positive 
Thompson test and palpable gap on physical examination can be used to diagnose acute ATR clinically [23]. Even though ultrasound has become the imaging modality of choice in many clinical settings to assess tendinopathies, it is recommended that ultrasound be paired with MRI in atypical cases when full diagnosis cannot be reached [11] [23] [24].

Growing evidence has made it obvious that early intervention in patients with Achilles tendon rupture extensively affects the prognosis. With the recent technological advances, along with safety, cost-effective, and no associated radiation, there is a larger trend among doctors to integrate the use of US into their routine clinical assessment [7]. Given its advantages, US is a favored imaging modality by many physicians and is expected to be used in establishing an accurate diagnosis of tendinopathies.

\section{Conflicts of Interest}

The authors declare no conflicts of interest regarding the publication of this paper.

\section{References}

[1] Pedowitz, D. and Kirwan, G. (2013) Achilles Tendon Ruptures. Current Reviews in Musculoskeletal Medicine, 6, 285-293. https://doi.org/10.1007/s12178-013-9185-8

[2] Moore, K., Agur, A., Dalley, A. and Moore, K. (2013) Clinically Oriented Anatomy. 7th Edition, Lippincott, Philadelphia, 596-597.

[3] Egger, A.C. and Berkowitz, M.J. (2017) Achilles Tendon Injuries. Current Reviews in Musculoskeletal Medicine, 10, 72-80. https://doi.org/10.1007/s12178-017-9386-7

[4] Maughan, M.D.K., Boggess, D.O. and Faafp, B. (2018) Achilles Tendinopathy and Tendon Rupture.

https://www.uptodate.com/contents/achilles-tendinopathy-and-tendon-rupture?sea $\underline{\mathrm{rch}=\text { achilles\%20tendon\%20rupture\&source=search_result\&selectedTitle }=1 \sim 16 \& \text { us }}$ age_type $=$ default $\&$ display_rank $=1$

[5] Bruyn, G.A.W. (2017) Musculoskeletal Ultrasonography: Clinical Application. https://www.uptodate.com/contents/musculoskeletal-ultrasonography-clinical-applica tions?search=ultrasound\%20in\%20achilles\%20tendon\%20rupture\&source=search_res ult\&selectedTitle $=2 \sim 150 \&$ usage_type $=$ default\&display_rank $=2$

[6] Stickles, S.P., Friedman, L., Demarest, M. and Raio, C. (2015) Achilles Tendon Rupture. The Western Journal of Emergency Medicine, 16, 161-162. https://doi.org/10.5811/westjem.2014.10.24127

[7] Patil, P. and Dasgupta, B. (2012) Role of Diagnostic Ultrasound in the Assessment of Musculoskeletal Diseases. Therapeutic Advances in Musculoskeletal Disease, 4, 341-355. https://doi.org/10.1177/1759720X12442112

[8] Weinreb, J.H., Sheth, C., Apostolakos, J., et al. (2014) Tendon Structure, Disease, and Imaging. Muscle, Ligaments and Tendons Journal, 4, 66-73.

[9] (2019) Ultrasound National Institute of Biomedical Imaging and Bioengineering. Nibib.nih.gov. https://www.nibib.nih.gov/science-education/science-topics/ultrasound

[10] Peck, J. and Bahner, D. (2017) Diagnosis of Achilles Tendon Rupture (ATR) with US in ED Setting. 
[11] Kayser, R., Mahlfeld, K. and Heyde, C. (2005) Partial Rupture of the Proximal Achilles Tendon: A Differential Diagnostic Problem in Ultrasound Imaging. British Journal of Sports Medicine, 39, 838-842. https://doi.org/10.1136/bjsm.2005.018416

[12] Bahner, D., Peck, J. and Gustafson, K. (2017) Diagnosis of Achilles Tendon Rupture with Ultrasound in the Emergency Department Setting. International Journal of Academic Medicine, 3, 205. https://doi.org/10.4103/IJAM.IJAM_16_17

[13] Chang, A. and Miller, T. (2009) Imaging of Tendons. Sports Health: A Multidisciplinary Approach, 1, 293-300. https://doi.org/10.1177/1941738109338361

[14] Precerutti, M., Bonardi, M., Ferrozzi, G. and Draghi, F. (2013) Sonographic Anatomy of the Ankle. Journal of Ultrasound, 17, 79-87.

https://doi.org/10.1007/s40477-013-0025-x

[15] Hodgson, R., O'Connor, P. and Grainger, A. (2012) Tendon and Ligament Imaging. The British Journal of Radiology, 85, 1157-1172. https://doi.org/10.1259/bjr/34786470

[16] Lento, P. and Primack, S. (2007) Advances and Utility of Diagnostic Ultrasound in Musculoskeletal Medicine. Current Reviews in Musculoskeletal Medicine, 1, 24-31. https://doi.org/10.1007/s12178-007-9002-3

[17] Meyer, N., Jacobson, J., Kalia, V. and Kim, S. (2018) Musculoskeletal Ultrasound: Athletic Injuries of the Lower Extremity. Ultrasonography, 37, 175-189. https://doi.org/10.14366/usg.18013

[18] Fouré, A. (2016) New Imaging Methods for Non-Invasive Assessment of Mechanical, Structural, and Biochemical Properties of Human Achilles Tendon: A Mini Review. Frontiers in Physiology, 7, 324. https://doi.org/10.3389/fphys.2016.00324

[19] Nadeau, M., Desrochers, A., Lamontagne, M., Larivière, C. and Gagnon, D. (2016) Quantitative Ultrasound Imaging of Achilles Tendon Integrity in Symptomatic and Asymptomatic Individuals: Reliability and Minimal Detectable Change. Journal of Foot and Ankle Research, 9, 30. https://doi.org/10.1186/s13047-016-0164-3

[20] Kälebo, P., Goksör, L.A., Swärd, L. and Peterson, L. (1990) Soft-Tissue Radiography, Computed Tomography, and Ultrasonography of Partial Achilles Tendon Ruptures. Acta Radiologica, 31, 565-570. https://doi.org/10.1177/028418519003100606

[21] Czyrny, Z. (2017) Standards for Musculoskeletal Ultrasound. Journal of Ultrasonography, 17, 182-187. https://doi.org/10.15557/JoU.2017.0027

[22] Liu, W., Zhuang, H., Shao, D., Wang, L. and Shi, M. (2017) High-Frequency Color Doppler Ultrasound in Diagnosis, Treatment, and Rehabilitation of Achilles Tendon Injury. Medical Science Monitor, 23, 5752-5759. https://doi.org/10.12659/MSM.904186

[23] Westin, O., Nilsson, H.K., Grävare, S.K., Möller, M., Kälebo, P. and Karlsson, J. (2016) Acute Ultrasonography Investigation to Predict Reruptures and Outcomes in Patients with an Achilles Tendon Rupture. Orthopaedic Journal of Sports Medicine, 4, 232596711666792. https://doi.org/10.1177/2325967116667920

[24] Garras, D.N., Raikin, S.M., Bhat, S.B., Taweel, N. and Karanjia, H. (2012) MRI Is Unnecessary for Diagnosing Acute Achilles Tendon Ruptures: Clinical Diagnostic Criteria. Clinical Orthopaedics and Related Research, 470, 2268-2273. https://doi.org/10.1007/s11999-012-2355-y 